\title{
Diagnostic performance of PET in thyroid cancer with elevated anti-Tg Ab
}

\author{
Seong-Jang Kim,2,3, Sang-Woo Lee ${ }^{4,5}$, Kyoungjune Pak ${ }^{6}$ and Sung-Ryul Shim \\ 1Department of Nuclear Medicine, Pusan National University Yangsan Hospital, Yangsan, Korea \\ 2BioMedical Research Institute for Convergence of Biomedical Science and Technology, Pusan National University Yangsan Hospital, Yangsan, Korea \\ ${ }^{3}$ Department of Nuclear Medicine, College of Medicine, Pusan National University, Yangsan, Korea \\ ${ }^{4}$ Department of Nuclear Medicine, School of Medicine, Kyungpook National University, Daegu, Korea \\ ${ }^{5}$ Department of Nuclear Medicine, Kyungpook National University Hospital, Daegu, Korea \\ ${ }^{6}$ Department of Nuclear Medicine, Pusan National University Hospital, Busan, Korea \\ ${ }^{7}$ Institute for Clinical Molecular Biology Research, Soonchunhyang University Hospital, Soonchunhyang University College of Medicine, Seoul, Korea \\ Correspondence should be addressed to S-J Kim: growthkim@pusan.ac.kr
}

\begin{abstract}
We aimed to explore the role of the diagnostic accuracy of F-18 fluorodeoxyglucose positron emission tomography/computed tomography (PET/CT) for the detection of recurrent and/or metastatic diseases in differentiated thyroid cancer (DTC) patients with progressively and/or persistently elevated $\mathrm{TgAb}$ levels and negative radioactive iodine whole-body scan (RI-WBS) through a systematic review and meta-analysis. The MEDLINE, EMBASE and Cochrane Library database, from the earliest available date of indexing through June 30, 2017, were searched for studies evaluating the diagnostic performance of F-18 FDG PET/CT for the detection of recurrent and/or metastatic diseases in DTC patients with progressively and/or persistently elevated TgAb levels and negative RI-WBS. We determined the sensitivities and specificities across studies, calculated positive and negative likelihood ratios (LR+ and LR-). Across 9 studies (515 patients), the pooled sensitivity for F-18 FDG PET/CT was $0.84(95 \% \mathrm{Cl} ; 0.77-0.89)$ a pooled specificity of 0.78 (95\% Cl; 0.67-0.86). Likelihood ratio (LR) syntheses gave an overall positive likelihood ratio (LR+) of $3.8(95 \% \mathrm{Cl} ; 2.5-5.7)$ and negative likelihood ratio (LR-) of $0.21(95 \% \mathrm{Cl}$; $0.14-0.30)$. The pooled diagnostic odds ratio (DOR) was 18 (95\% Cl; 10-34). The area (AUC) under the hierarchical summary receiver-operating characteristic (HCROC) curve was 0.88 (95\% Cl: 0.85-0.90). F-18 FDG PET or PET/CT demonstrated moderate sensitivity and specificity for the detection of recurrent and/or metastatic diseases in DTC patients with progressively and/or persistently elevated TgAb levels and negative RI-WBS.
\end{abstract}

\author{
Key Words \\ - positron emission \\ tomography \\ - PET/CT \\ - thyroid neoplasm \\ - anti-thyroglobulin antibody
}

\section{Introduction}

Differentiated thyroid cancers (DTC) account for $1 \%$ of all malignant tumors and are the most common malignant endocrine tumor. In the United States, an estimated 14,400 men and 42,470 women will be diagnosed with thyroid cancer and 920 men and 1090 women will die of the disease at 2017 (Siegel et al. 2017). Serum thyroglobulin (Tg) measurement and neck ultrasonography are considered to be the mainstays of postoperative follow-up in DTC patients (Haugen et al. 2016). Also, Tg is an important biological marker of persistent and/or recurrent disease after total thyroidectomy and radioactive iodine-131 ablation (RIA) in DTC patients. 
Anti-thyroglobulin autoantibodies ( $\mathrm{TgAb})$ are known to be more common in DTC patients, especially in papillary thyroid cancer (PTC) compared with the general population (Kumar et al. 1994, Souza et al. 2003). Also, $\mathrm{TgAb}$ could interfere with serum $\mathrm{Tg}$ measurement and compromises the clinical utility of Tg monitoring of DTC patients for recurrence (Spencer et al. 2005, 2011, Kim et al. 2010). Theoretically, TgAb might be produced as a result of presence of thyroid tissue presence. Therefore, increasing serum $\mathrm{TgAb}$ levels during the follow-up of DTC patients appear to be a good biomarker for persistent or recurrent DTC patients with undetectable serum Tg levels. Some previous studies demonstrated these relationships in DTC patients (Tsushima et al. 2013, Durante et al. 2014, Hsieh \& Wang 2014). On the contrary, some other studies could not find these phenomenon (Kumar et al. 1994, Görges et al. 2005).

F-18 fluorodeoxyglucose (FDG) positron emission tomography/computed tomography (PET/CT) is a functional imaging tool for the detection, staging and management of various cancers. F-18 FDG PET/CT has emerged as an important tool for the management of patients with DTC and is most valuable in patients with increasing $\mathrm{Tg}$ levels with a negative radioactive iodine whole-body scan (RI-WBS) after RIA (Joensuu \& Ahonen 1987, Abraham \& Schöder 2011). According to the ATA guidelines, F-18 FDG PET/CT should be performed only when unstimulated $\mathrm{Tg}$ levels exceed $10 \mathrm{ng} / \mathrm{mL}$ (ATA Taskforce 2009).

Several previous studies have evaluated the diagnostic accuracy of F-18 FDG PET or PET/CT for the detection of recurrent and/or metastatic diseases in DTC patients with negative RI-WBS and elevated TgAb level with variable diagnostic accuracy (Seo et al. 2010, Ozkan et al 2012, 2013, Qiu et al. 2017). The purpose of the current study is to meta-analyze the published data on the diagnostic accuracy of F-18 FDG PET or PET/CT for the detection of recurrent and/or metastatic diseases in DTC patients with progressively and/or persistently elevated TgAb levels and negative RI-WBS.

\section{Materials and methods}

\section{Data sources and search strategy}

We conducted electronic English-language literature searches of MEDLINE via PubMed, EMBASE and Cochrane Library database from the earliest available date of indexing through June 30, 2017. We also handsearched the reference lists of identified publications for additional studies. We used a search algorithm based on a combination of terms: (1) 'PET' OR 'positron emission tomography' OR 'positron emission tomography/ computed tomography' OR 'PET/CT' 'positron emission tomography-computed tomography' OR 'PET-CT'; and (2) 'Thyroid neoplasms' OR 'Thyroid cancer' OR 'Papillary thyroid cancer' OR 'Follicular thyroid cancer' and (3) 'Anti-thyroglobulin antibody' OR 'Anti-TG'.

\section{Study selection}

The inclusion criteria for relevant studies were as follows: whole-body F-18 FDG PET or PET/CT had been used to identify the recurrent and/or metastatic diseases in patients with elevated $\mathrm{TgAb}$ and negative RI-WBS; sufficient data to reassess sensitivity and specificity of F-18 FDG PET or PET/CT or absolute numbers of true-positive, true-negative, false-positive and false-negative data had been presented; and no data overlap.

Duplicate publications were excluded, as were publications such as review articles, case reports, conference papers and letters, which do not contain the original data. Two researchers independently reviewed titles and abstracts of the retrieved articles, applying the above-mentioned selection criteria. Articles were rejected if clearly ineligible. The same four researchers then independently evaluated the full-text version of the included articles to determine their eligibility for inclusion.

\section{Data extraction and quality assessment}

Information about basic study (authors, year of publication and country of origin), study design (prospective or retrospective), patients' characteristics and technical aspects were collected.

Each study was analyzed to retrieve the number of true-positive (TP), true-negative (TN), false-positive (FP) and false-negative (FN) findings of F-18 FDG PET or PET/CT for the detection of recurrent and/or metastatic diseases in patients with elevated $\operatorname{TgAb}$ and negative RI-WBS, according to the reference standard. Only studies providing such complete information were finally included in the meta-analysis.

Quality of the included studies was assessed based on 15-item modified Quality Assessment of Diagnostic Accuracy Studies (QUADAS2) (Whiting et al. 2011). Two reviewers independently assessed each potentially eligible study and assigned them as a quality rating of 'good,' 'fair' or 'poor'. Quality assessment was conducted 
based on following criteria: study design and presence of bias including selection, performance, recording and reporting bias. Studies with high risk of bias were defined as poor quality, presence of moderate risk (did not affect the results) as fair quality, and those with minimal risk as good quality. Disagreements were settled with consensus decision. Disagreement between the 2 authors was resolved by discussion.

\section{Data synthesis and analysis}

All data from each eligible study were extracted. Categorical variables are presented as frequencies or percentages, and continuous variables are presented as mean values unless stated otherwise. Measures of the diagnostic performance, including sensitivity, specificity and diagnostic odds ratios (DORs), are reported as point estimates with 95\% confidence intervals (CIs). A DOR can be calculated as the ratio of the odds of positivity in a disease state relative to the odds of positivity in the non-disease state, with higher values indicating better discriminatory test performance (Glas et al. 2003). Between-study statistical heterogeneity was assessed using $I^{2}$ and the Cochrane $Q$ test on the basis of the random-effects analysis (Thompson 1994). Publication bias was examined using the effective sample size funnel plot and associated regression test of asymmetry described by Deeks et al. (2005). We used the bivariate random-effects model for analysis and pooling of the diagnostic performance measures across studies, as well as comparisons between different index tests (Reitsma et al. 2005, Hamza et al. 2008). The bivariate model estimates pairs of logit transformed sensitivity and specificity from studies, incorporating the correlation that might exist between sensitivity and specificity. We also used the model to create hierarchical summary receiver-operating characteristic curves and to estimate the area under the curve (Rutter \& Gatsonis 2001). When statistical heterogeneity was substantial, we performed meta-regression to identify potential sources of bias (Lijmer et al. 1999) using freeware of Meta-disc (Meta-DiSc, version 1.4, Universidad Complutense, Madrid, Spain). Meta-regression was performed to examine the potential source of the heterogeneity and the covariates were (1) ethnicity (Asian vs Caucasian), (2) detection device (PET vs PET/CT), (3) cancer type (PTC vs PTC and FTC) and (4) titer of $\mathrm{TgAb}$ (Continuous variable).

Pooled estimates were also calculated for subgroups of studies that were defined according to specific study designs. Two-sided $P \leq 0.05$ was considered statistically significant. Statistical analyses were performed with midas command in commercial software programs (STATA, version 13.1; StataCorp LP).

\section{Ethical approval}

Institutional review board approval was not required because we only performed data analysis based on the published studies.

Table 1 Characteristics of the included studies.

\begin{tabular}{|c|c|c|c|c|c|c|c|c|c|c|c|}
\hline Authors (year) & Country & Device & Analysis & $\begin{array}{l}\text { Patient } \\
\text { number }\end{array}$ & $\begin{array}{l}\text { Cancer } \\
\text { type }\end{array}$ & Age & Male/female & $\begin{array}{c}\text { F-18 FDG } \\
\text { dose }(\mathrm{MBq})\end{array}$ & $\begin{array}{c}\text { Mean TgAb } \\
\text { (IU/mL, range) }\end{array}$ & $\begin{array}{l}\text { TgAb } \\
\text { measure }\end{array}$ & $\begin{array}{l}\text { Study } \\
\text { design }\end{array}$ \\
\hline $\begin{array}{c}\text { Asa et al. } \\
\text { (2014) }\end{array}$ & Turkey & $\mathrm{PET} / \mathrm{CT}$ & PB & 40 & $\begin{array}{l}\text { PTC } \\
\text { FTC }\end{array}$ & 43.1 & $8 / 32$ & NA & $1101.2(150-4000)$ & ECLIA & $\mathrm{R}$ \\
\hline $\begin{array}{l}\text { Bogsrud et al. } \\
\text { (2011) }\end{array}$ & USA & PET & PB & 17 & $\begin{array}{l}\text { PTC } \\
\text { FTC }\end{array}$ & 51.5 & $9 / 8$ & 740 & $416(10-6500)$ & ECLIA & $\mathrm{R}$ \\
\hline $\begin{array}{l}\text { Chung et al. } \\
\text { (2002) }\end{array}$ & Korea & PET & PB & 26 & PTC & 41.1 & $1 / 25$ & 370 & $983.2(25-7439)$ & RIA & $\mathrm{R}$ \\
\hline $\begin{array}{l}\text { Kingpetch } \\
\text { et al. (2011) }\end{array}$ & Thai & $\mathrm{PET} / \mathrm{CT}$ & PB & 22 & $\begin{array}{l}\text { PTC } \\
\text { FTC }\end{array}$ & NA & NA & $227.5-455.4$ & 414.6 & RIA & $\mathrm{R}$ \\
\hline $\begin{array}{l}\text { Ozkan et al. } \\
\text { (2012) }\end{array}$ & Turkey & $\mathrm{PET} / \mathrm{CT}$ & PB & 31 & PTC & 50.1 & $4 / 27$ & 370 & $472.9(27-4000)$ & RIA & $\mathrm{R}$ \\
\hline $\begin{array}{l}\text { Ozkan et al. } \\
\text { (2013) }\end{array}$ & Turkey & $\mathrm{PET} / \mathrm{CT}$ & PB & 10 & PTC & NA & NA & 370 & $663.4(71.3-4000)$ & RIA & $\mathrm{R}$ \\
\hline $\begin{array}{l}\text { Qiu et al. } \\
\text { (2017) }\end{array}$ & China & $\mathrm{PET} / \mathrm{CT}$ & PB & 82 & PTC & 48 & $32 / 50$ & $3-4 \mathrm{MBq} / \mathrm{kg}$ & 479 (98-3726) & ECLIA & $\mathrm{R}$ \\
\hline $\begin{array}{l}\text { Viedma et al. } \\
\text { (2011) }\end{array}$ & Spain & PET & LB & 11 & PTC & NA & NA & $370-434$ & 200 & ECLIA & $\mathrm{R}$ \\
\hline $\begin{array}{l}\text { Seo et al. } \\
(2010)\end{array}$ & Korea & $\mathrm{PET} / \mathrm{CT}$ & PB & 276 & $\begin{array}{l}\text { PTC } \\
\text { FTC }\end{array}$ & NA & NA & $370-555$ & NA & RIA & $\mathrm{R}$ \\
\hline
\end{tabular}

ECLIA, electrochemiluminescence immunoassay; FTC, follicular thyroid cancer; LB, lesion-based; NA, not available; PB, patient based; PTC, papillary thyroid cancer; $\mathrm{R}$, retrospective; RIA, radioimmunoassay. 


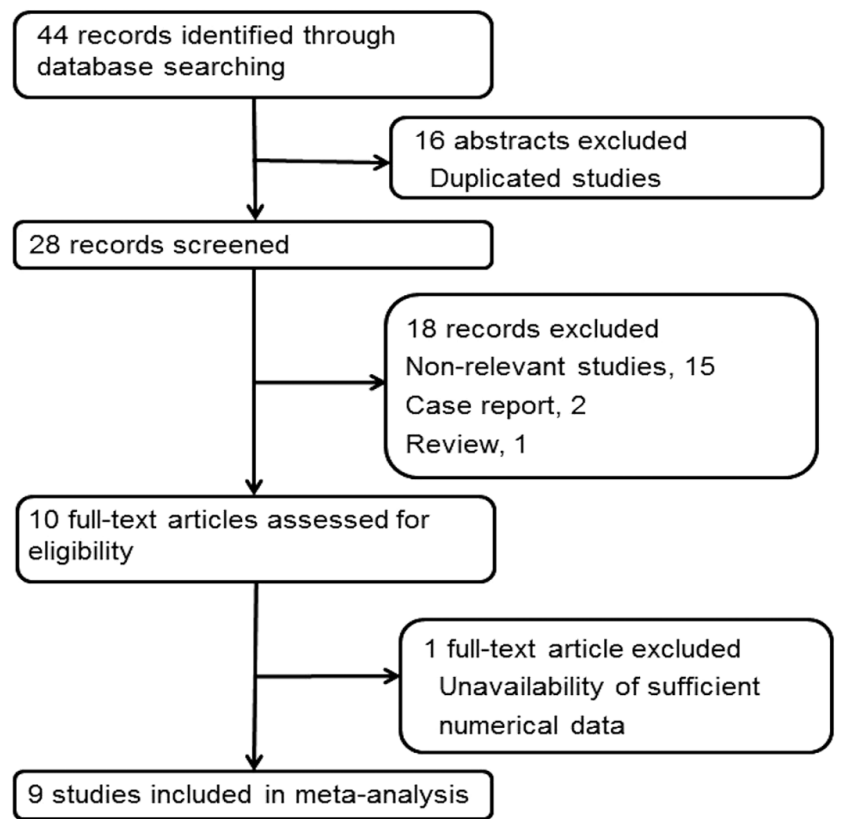

Figure 1

Flow chart of the search for eligible studies on the diagnostic performance of F-18 FDG PET or PET/CT for detection of recurrent and/or metastatic diseases in DTC patients with progressively and/or persistently elevated TgAb levels and negative RI-WBS.

\section{Results}

\section{Literature search and selection of studies}

After the comprehensive computerized search was performed and references lists were extensively crosschecked, our research yielded 44 records, of which 16 duplicated records were excluded after reviewing the title and abstract. Also, non-relevant 15 abstracts, 2 case reports, 1 review article were excluded. Remaining 10 full-text articles were assessed for eligibility and 1 article was excluded due to unavailability of sufficient numerical data $(n=9)$ for the calculation of sensitivity and specificity of F-18 FDG PET or PET/CT for the detection of recurrent and/or metastatic diseases in DTC patients with progressively and/or persistently elevated $\mathrm{TgAb}$ levels and negative RI-WBS. Finally, 9 studies were selected and were eligible for the systematic review and meta-analysis, and no additional studies were found screening the references of these articles (Chung et al. 2002, Seo et al. 2010, Bogsrud et al. 2011, Kingpetch et al . 2011, Viedma et al. 2011, Ozkan et al. 2012, 2013, Asa et al. 2014, Qiu et al. 2017). The characteristics of the included studies are presented in Table 1. The detailed procedure of study selection in the meta-analysis is shown in Fig. 1.

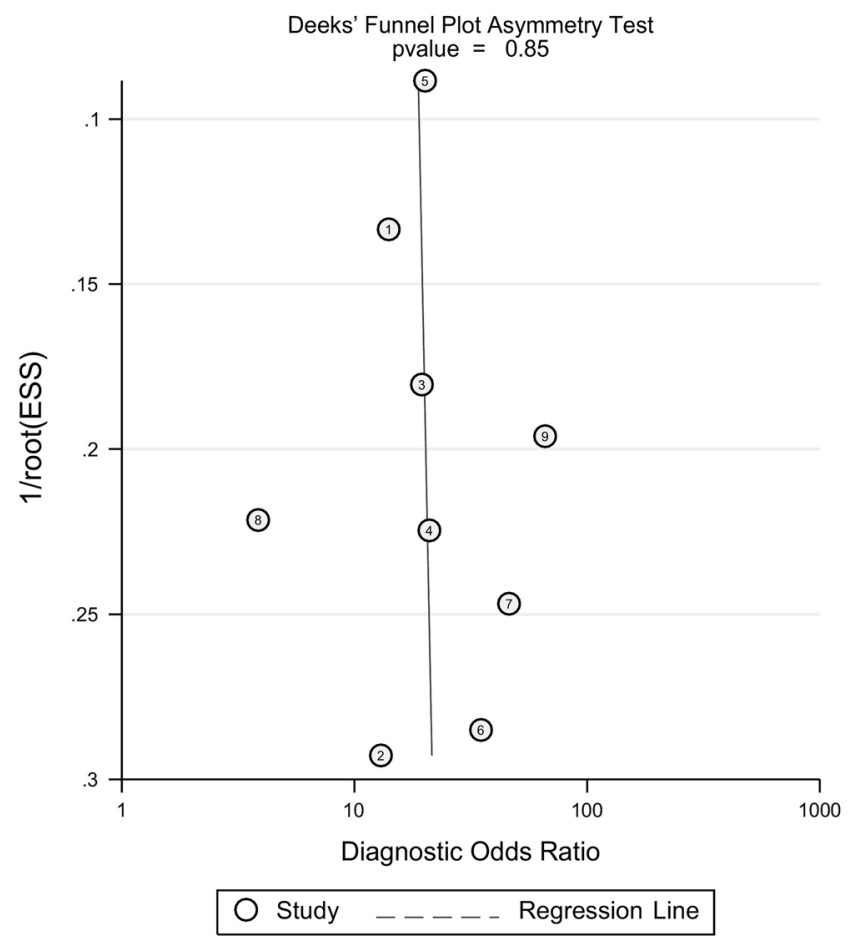

Figure 2

Results of Deeks's funnel plot of asymmetry test for publication bias. Non-significant slope indicates that no significant bias was found. ESS effective sample size. 1, Qiu et al. 2017; 2, Ozkan et al. 2013; 3, Ozkan et al. 2012; 4, Kingpetch et al. 2011; 5, Seo et al. 2010; 6, Viedma et al. 2011; 7, Bogsrud et al. 2011; 8, Asa et al. 2014; 9, Chung et al. 2002.

\section{Study description, quality and publication bias}

We conducted all analyses based on per-patient data and/or per-lesion data analysis. Among those 9 studies included in the current review, 8 studies conducted patient-based analysis of F-18 FDG PET/CT (Chung et al. 2002, Seo et al. 2010, Bogsrud et al. 2011, Kingpetch et al. 2011, Ozkan et al. 2012, 2013, Asa et al. 2014, Qiu et al. 2017). The other study conducted lesionbased analysis (Viedma et al. 2011). There were a total of 515 patients in the included studies, and the age ranged from 17 to 82 years. A total 50 patients were male, and 142 patients were female. The four studies did not report the number of male and female patients in their population (Seo et al. 2010, Kingpetch et al. 2011, Viedma et al. 2011, Ozkan et al. 2013). Of all 9 studies enrolled patients retrospectively. Six studies used PET/CT (Seo et al. 2010, Kingpetch et al. 2011, Ozkan et al. 2012, 2013, Asa et al. 2014, Qiu et al. 2017) and other studies (Chung et al. 2002, Bogsrud et al. 2011, Viedma et al. 2011) used PET as imaging device in their studies. The principal characteristics of the 9 studies 


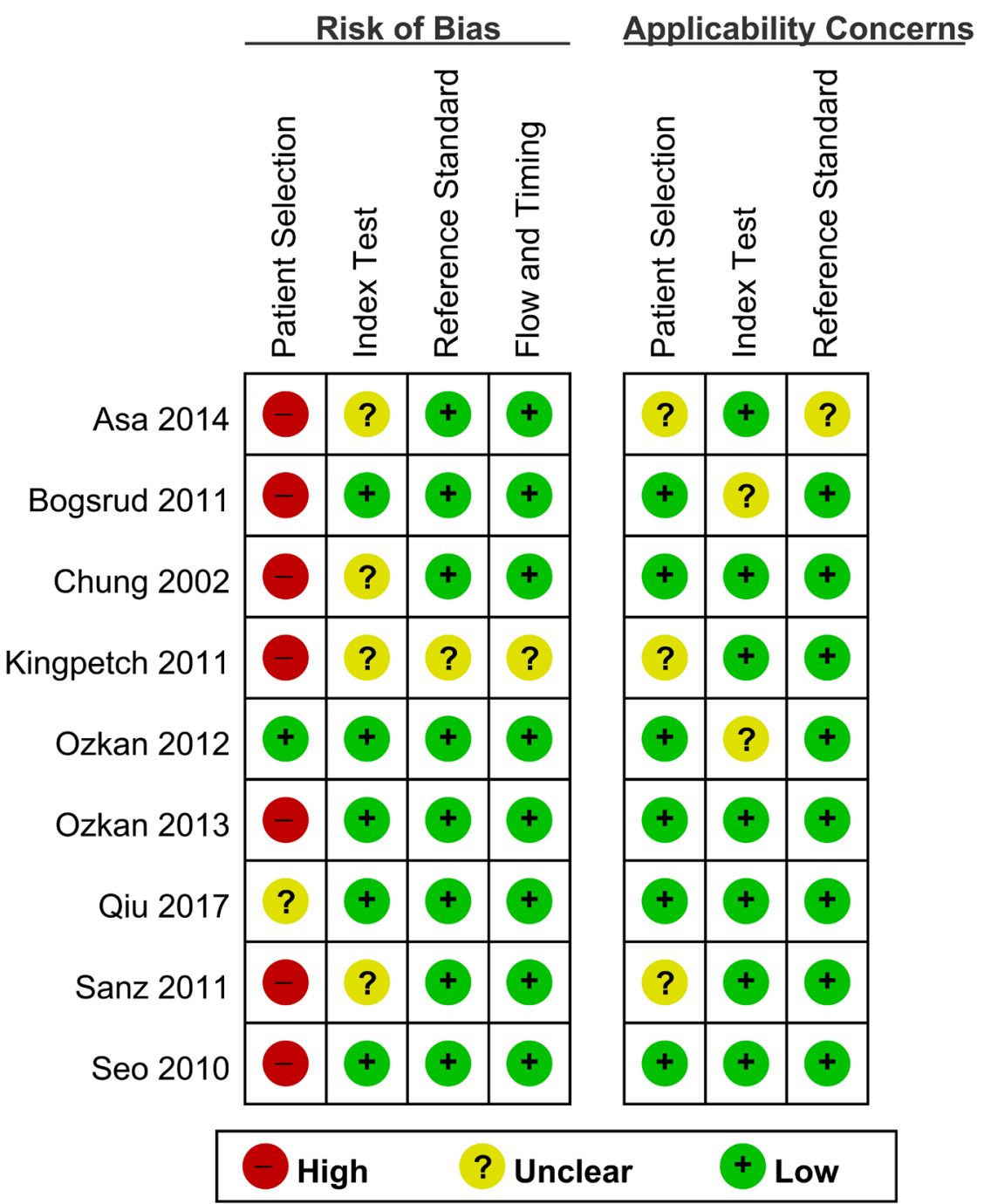

Figure 3

Risk of bias and applicability concerns summary.

included in the meta-analysis are included in Table 1. To assess a possible publication bias, Deeks's funnel plot asymmetry tests were designed. The non-significant slope indicates that no significant bias was found. The $P$ value was 0.85 (Fig. 2).

\section{Methodological quality assessment}

Figure 3 shows the risk of bias and applicability concerns summary and overall, the quality of the included studies was deemed satisfactory.

\section{Diagnostic accuracy of F-18 FDG PET or PET/CT}

The diagnostic performance results of F-18 PET/CT of the 9 included studies in the meta-analysis are presented in Fig. 4 and Table 2. The pooled sensitivity for F-18 FDG PET/CT was 0.84 (95\% CI; 0.77-0.89) without heterogeneity
$\left(I^{2}=0.00,95 \% \mathrm{CI}=0.00-100.00\right)$ and a pooled specificity of 0.78 (95\% CI; 0.67-0.86) with heterogeneity $\left(I^{2}=57.63\right.$, 95\% CI=26.25-89.02). LR syntheses gave an overall positive likelihood ratio (LR+) of 3.8 (95\% CI; $2.5-5.7)$ and negative likelihood ratio (LR-) of 0.21 (95\% CI; 0.14-0.30). The pooled DOR was 18 (95\% CI; 10-34). Forest plots of the sensitivity and specificity of F-18 FDG PET/CT for the detection of recurrent and metastatic lesions are shown in Fig. 4. Figure 5 shows hierarchical summary receiveroperating characteristic (ROC) curve and indicates that the areas under the curve was 0.88 (95\% CI; 0.85-0.90), indicating moderate diagnostic accuracy.

\section{Heterogeneity evaluation and meta-regression analysis}

Between-study heterogeneity was present for specificity among studies of F-18 FDG PET or PET/CT for the 

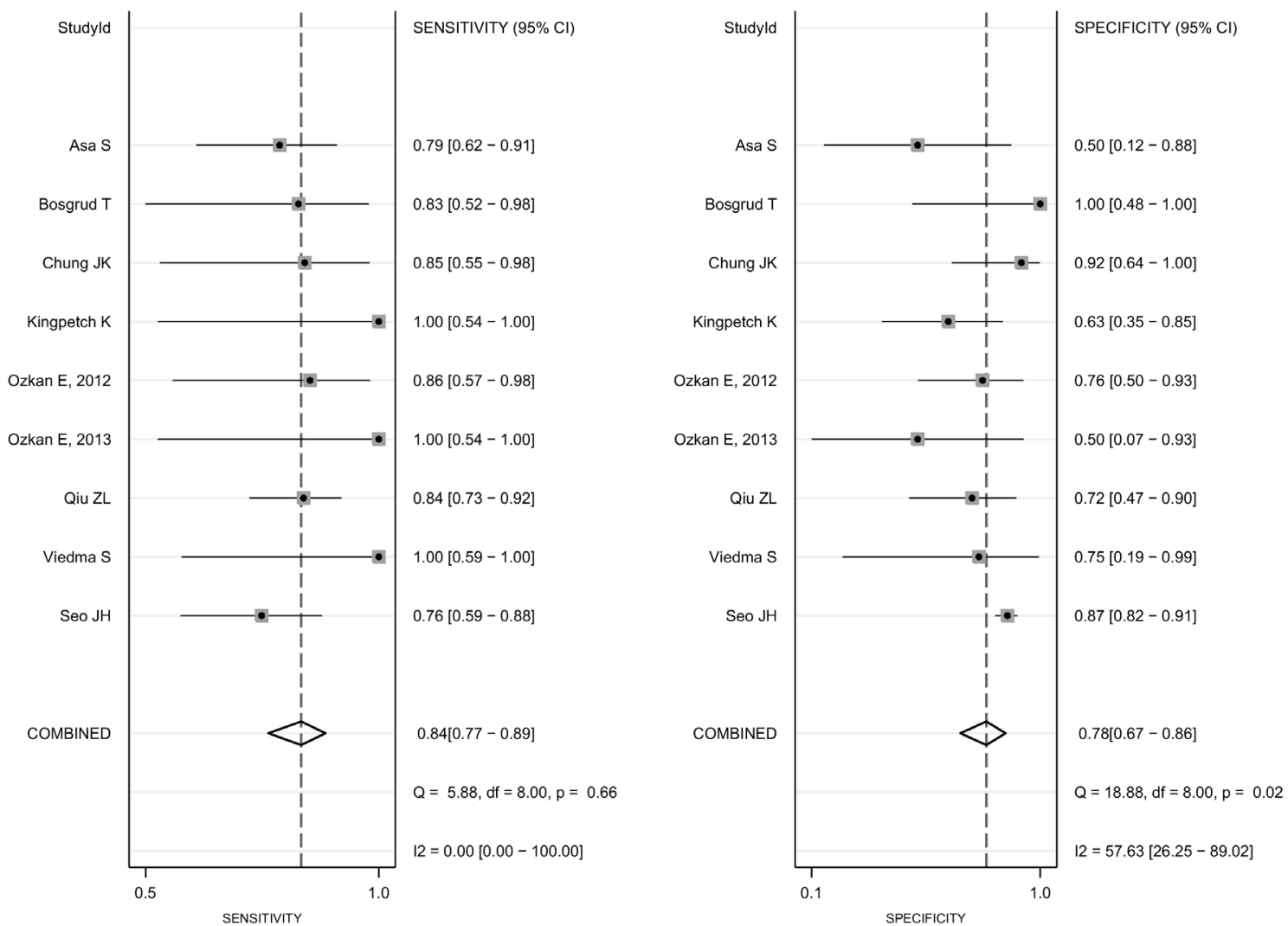

Figure 4

Forest plot of pooled sensitivity and specificity of F-18 FDG PET or PET/CT for detection of recurrent and/or metastatic diseases in DTC patients with progressively and/or persistently elevated TgAb levels and negative RI-WBS. Summary of sensitivity and specificity of F-18 FDG PET or PET/CT was 0.84 (95\% confidence interval (Cl); 0.77-0.89) and $0.78(95 \% \mathrm{Cl} ; 0.67-0.86)$, respectively. (Q; Cohran's $Q, 12 ; 12$ is the percentage of observed total variation between studies.)

detection of recurrent and/or metastatic diseases. A meta-regression analysis was performed to explore other sources of heterogeneity in the studies of F-18 FDG PET or PET/CT. Meta-regression showed that the device (PET vs PET/CT) was the major source of heterogeneity in the meta-analysis (Table 3).

\section{Discussion}

It is known that persistent and/or a rising TgAb level after total thyroidectomy and RIA is a risk factor of recurrent/persistent disease of DTC (Seo et al. 2010, Hsieh \& Wang 2014). Also, elevated $\operatorname{TgAb}$ may be alternative biological marker of the recurrent and/or metastatic disease (Seo et al. 2010). Durante et al. reported that PTC patients with positive serum TgAb titer during the first year after primary treatment were more likely to have persistent/recurrent disease than those who were consistently TgAb-negative and negative titers at 1 year may be associated with more favorable outcomes (Durante et al. 2014). Also, Kim et al. demonstrated that serum TgAb levels measured at 6-12 months after remnant ablation could predict recurrence in patients with undetectable $\mathrm{Tg}$ values (Kim et al. 2008). In patients with undetectable $\mathrm{Tg}$ and positive $\mathrm{TgAb}$ values, a change in $\mathrm{TgAb}$ concentration during the early postoperative period may be a prognostic indicator of recurrence (Kim et al. 2008).

F-18 FDG PET/CT is used in the postoperative surveillance of DTC, mainly after RIA in patients with elevated serum $\mathrm{Tg}$ levels but negative RI-WBS (ATA Taskforce 2009). A recent meta-analysis of the diagnostic value of F-18 FDG PET or PET/CT in DTC patients who showed with elevated serum $\mathrm{Tg}$ post-thyroidectomy and negative RI-WBS demonstrated that these imaging techniques had a good diagnostic accuracy, with pooled sensitivity and specificity values of 88.5 and $84.7 \%$ respectively (Dong et al. 2009). They concluded that F-18 FDG PET is especially effective in detecting patients with elevated Tg levels and normal RI-WBS and F-18 FDG PET/CT is a more sensitive method in the follow-up of DTC recurrence or metastases, particularly in those with negative RI-WBS (Dong et al. 2009). 
Table 2 Diagnostic performance of F-18 FDG PET or PET/CT for the detection of recurrent and/or metastatic diseases in differentiated thyroid cancer patients with elevated $\mathrm{TgAb}$ and negative radioactive iodine whole-body scan.

\begin{tabular}{|c|c|c|c|c|c|c|c|c|}
\hline \multirow[b]{2}{*}{ Authors (year) } & \multicolumn{4}{|c|}{ Test results, number of patients or lesions } & \multicolumn{4}{|c|}{$95 \% \mathrm{Cl}$} \\
\hline & $\begin{array}{l}\text { True } \\
\text { positive }\end{array}$ & $\begin{array}{c}\text { False } \\
\text { positive }\end{array}$ & $\begin{array}{c}\text { False } \\
\text { negative }\end{array}$ & $\begin{array}{c}\text { True } \\
\text { negative }\end{array}$ & Sensitivity & Specificity & PPV & NPV \\
\hline$\overline{\text { Asa et al. (2014) }}$ & 27 & 3 & 7 & 3 & $0.79(0.62-0.91)$ & $0.50(0.12-0.88)$ & $0.9(0.72-0.97)$ & $0.3(0.08-0.65)$ \\
\hline $\begin{array}{l}\text { Bogsrud et al. } \\
\text { (2011) }\end{array}$ & 10 & 0 & 2 & 5 & $0.83(0.52-0.98)$ & $1.00(0.48-1.00)$ & $1.0(0.66-1.00)$ & $0.71(0.30-0.95)$ \\
\hline $\begin{array}{l}\text { Chung et al. } \\
\text { (2002) }\end{array}$ & 11 & 1 & 2 & 12 & $0.85(0.55-0.98)$ & $0.92(0.64-1.00)$ & $0.92(0.59-0.99)$ & $0.86(0.56-0.97)$ \\
\hline $\begin{array}{l}\text { Kingpetch et al. } \\
\text { (2011) }\end{array}$ & 6 & 6 & 0 & 10 & $1.00(0.54-1.00)$ & $0.63(0.35-0.85)$ & $0.5(0.22-0.78)$ & $1.0(0.66-1.00)$ \\
\hline $\begin{array}{l}\text { Ozkan et al. } \\
\text { (2012) }\end{array}$ & 12 & 4 & 2 & 13 & $0.86(0.57-0.98)$ & $0.76(0.50-0.93)$ & $0.75(0.47-0.92)$ & $0.87(0.58-0.98)$ \\
\hline $\begin{array}{l}\text { Ozkan et al. } \\
\text { (2013) }\end{array}$ & 6 & 2 & 0 & 2 & $1.00(0.54-1.00)$ & $0.50(0.07-0.93)$ & $0.75(0.36-0.96)$ & $1.0(0.19-1.00)$ \\
\hline Qiu et al. (2017) & 54 & 5 & 10 & 13 & $0.84(0.73-0.92)$ & $0.72(0.47-0.90)$ & $0.92(0.81-0.97)$ & $0.57(0.34-0.76)$ \\
\hline $\begin{array}{l}\text { Viedma et al. } \\
\text { (2011) }\end{array}$ & 7 & 1 & 0 & 3 & $1.00(0.59-1.00)$ & $0.75(0.19-0.99)$ & $0.88(0.47-0.99)$ & $1.0(0.31-1.00)$ \\
\hline Seo et al. (2010) & 28 & 32 & 9 & 207 & $0.76(0.59-0.88)$ & $0.87(0.82-0.91)$ & $0.47(0.34-0.59)$ & $0.96(0.92-0.98)$ \\
\hline Combined & 161 & 54 & 32 & 268 & $0.84(0.77-0.89)$ & $0.78(0.67-0.86)$ & $0.75(0.68-0.80)$ & $0.89(0.85-0.92)$ \\
\hline
\end{tabular}

$\mathrm{Cl}$, confidence interval; NPV, negative predictive value; PPV, positive predictive value.

However, few studies reported the clinical usefulness of F-18 FDG PET or PET/CT for the detection of recurrent and/or metastatic diseases in DTC patients with elevated serum $\mathrm{TgAb}$ and negative RI-WBS after total thyroidectomy and RIA. According to the current systematic review and meta-analysis, F-18 FDG PET or PET/CT has moderate diagnostic accuracy for the detection of recurrent and/or metastatic diseases in these patients group with an area under the ROC curve of 0.88 (95\% CI; 0.85-0.90). F-18 FDG PET or PET/CT demonstrated a sensitivity of 0.84 (95\% CI; 0.77-0.89) and a specificity of 0.78 (95\% CI; 0.67-0.86). A recent study by Qiu et al. investigated the diagnostic accuracy of F-18 FDG PET/CT for detection of recurrent and/or metastatic diseases in DTC patients with elevated serum $\mathrm{TgAb}$ and negative RI-WBS and reported $84 \%$ sensitivity, $72 \%$ specificity, $92 \%$ positive predictive value (PPV), $57 \%$ negative predictive value (NPV) and $82 \%$ accuracy (Qiu et al. 2017). They suggested that F-18 FDG PET/ CT should be performed routinely in PTC patients with negative $\mathrm{Tg}$, negative I-131 WBS at first post-ablation and progressively increased $\mathrm{TgAb}$ level, especially when span for progressively increased $\mathrm{TgAb}$ level $\geq 3$ years and/or progressively increased TgAb value up to $150 \mathrm{IU} / \mathrm{mL}$ (Qiu et al. 2017). Chung et al. first investigated the clinical usefulness of F-18 FDG PET in patients elevated serum TgAb levels (Chung et al. 2002). In their study, F-18 FDG PET showed a sensitivity, specificity and accuracy of 84.6, 92.3 and $88.4 \%$, respectively (Chung et al. 2002). Asa et al. investigated the diagnostic usefulness of F-18 FDG PET/CT in 40 patients with negative Tg and persistent/progressive elevated $\mathrm{TgAb}$ titers (Asa et al. 2014). On the basis of the total 16 patients with available final diagnoses, the sensitivity, specificity, PPV,

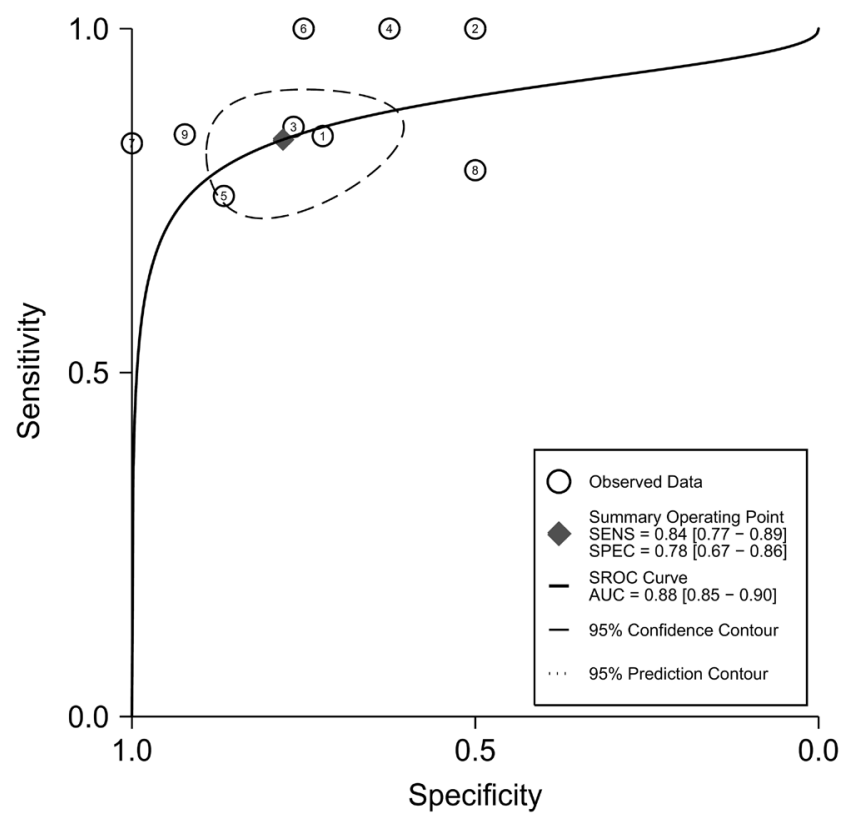

Figure 5

Hierarchical summary receiver-operating characteristic (HSROC) curves of F-18 FDG PET or PET/CT the detection of recurrent and/or metastatic diseases in DTC patients with progressively and/or persistently elevated TgAb levels and negative RI-WBS. 1, Qiu et al. 2017; 2, Ozkan et al. 2013; 3, Ozkan et al. 2012; 4, Kingpetch et al. 2011; 5, Seo et al. 2010; 6, Viedma et al. 2011; 7, Bogsrud et al. 2011; 8, Asa et al. 2014; 9, Chung et al. 2002. 
Table 3 Effects of moderators.

\begin{tabular}{l} 
Variables \\
\hline Ethnicity (Asian vs Caucasian) \\
Device (PET vs PET/CT) \\
Cancer type (PTC vs PTC and FTC) \\
TgAb (continuous variable)
\end{tabular}

$\begin{array}{r}\text { Coef. }{ }^{*} \\ \hline-0.421 \\ -1.265 \\ 0.329 \\ -0.001 \\ \hline\end{array}$

\begin{tabular}{c}
\hline SE \\
\hline 0.234 \\
0.273 \\
0.220 \\
0.000 \\
\hline
\end{tabular}

\begin{tabular}{c}
\hline DOR \\
\hline 0.660 \\
0.280 \\
1.390 \\
1.000 \\
\hline
\end{tabular}

\begin{tabular}{ll}
\hline \multicolumn{2}{c}{$95 \% \mathrm{Cl}$} \\
\hline 0.310 & 1.380 \\
0.120 & 0.670 \\
0.690 & 0.280 \\
1.000 & 1.000 \\
\hline
\end{tabular}

\begin{tabular}{c}
\hline $\boldsymbol{P t}^{+}$ \\
\hline 0.169 \\
0.019 \\
0.232 \\
0.089
\end{tabular}

Ethnicity (0, Asian vs 1, Caucasian); device (0, PET vs 1, PET/CT); cancer type (0, PTC vs 1, PTC and FTC).

* Regression coefficient; ${ }^{\dagger} P$ value of random effect meta-regression using maximum likelihood estimation (ML) between-study variances and the weighted least squares of study size for regression model estimation.

NPV and the accuracy were 78.5, 50, 91.6, 25 and 75\%, respectively (Asa et al. 2014). They concluded from the available data that F-18 FDG PET/CT can be effectively used in the detection of recurrence/metastasis in the follow-up of patients with DTC and negative serum Tg and a persistently/progressively increased TgAb level (Asa et al. 2014).

Interestingly, Chiovato et al. investigated whether complete removal of thyroid antigens results in the abatement of humoral thyroid autoimmunity. They showed that the persistence of thyroid peroxidase or thyroglobulin antibodies may be a marker of incomplete cure in many patients with differentiated thyroid carcinoma (Chiovato et al. 2003). Also, they concluded that the complete ablation of thyroid tissue with its antigenic components results in the disappearance of antibodies to all major thyroid antigens, thus supporting the concept that continued antibody production depends on the persistence of autoantigen in the body (Chiovato et al. 2003).

We should consider some limitations of the current meta-analysis. The inter-study heterogeneity was noticed in the current meta-analysis. The included studies were statistically heterogeneous in their estimates of specificity in the current study. We tried to overcome this limitation by using random-effects model that incorporates uncertainties arising because of inter-study variation, as well as performing meta-regression. To minimize bias in the selection of studies and in the data extraction, reviewers who were blinded to the journal, author, institution and date of publication independently selected articles based on the inclusion criteria, and scores were assigned to study design characteristics and examination results by using a standardized form that was based on the QUADAS2 tool. Also, publication bias is a major concern in all meta-analyses as studies reporting significant findings are more likely to be published than those reporting nonsignificant results. We assessed the publication bias in our analysis by using funnel plots, which showed very little evidence of asymmetry $(P=0.85)$.

\section{Conclusion}

F-18 FDG PET or PET/CT demonstrated moderate sensitivity and specificity for the detection of recurrent and/or metastatic diseases in DTC patients with progressively and/or persistently elevated TgAb levels and negative RI-WBS. At present, the literature regarding the use of F-18 FDG PET or PET/CT for this purpose remains still limited; thus, further large multicenter studies would be necessary to substantiate the diagnostic accuracy of F-18 FDG PET or PET/CT for the detection of recurrent and/or metastatic diseases in DTC patients with progressively and/or persistently elevated TgAb levels and negative RI-WBS.

\section{Declaration of interest}

The authors of this manuscript declare no relationships with any companies whose products or services may be related to the subject matter of the research reported.

\section{Funding}

This research did not receive any specific grant from any funding agency in the public, commercial or not-for-profit sector.

\section{Informed consent}

Written informed consent was not required for this study because it is a meta-analysis based on the studies that have been published.

\section{References}

Abraham T \& Schöder H 2011 Thyroid cancer-indications and opportunities for positron emission tomography/computed tomography imaging. Seminars in Nuclear Medicine 41 121-138. (https://doi.org/10.1053/j.semnuclmed.2010.10.006)

American Thyroid Association (ATA) Guidelines Taskforce on Thyroid Nodules and Differentiated Thyroid Cancer, Cooper DS, Doherty GM, Haugen BR, Kloos RT, Lee SL, Mandel SJ, Mazzaferri EL, McIver B, Pacini F, et al. 2009 Revised American Thyroid Association management guidelines for patients with thyroid nodules and differentiated thyroid cancer. Thyroid 19 1167-1214. (https://doi. org/10.1089/thy.2009.0110)

Asa S, Aksoy SY, Vatankulu B, Aliyev A, Uslu L, Ozhan M, Sager S, Halac M \& Sonmezoglu K 2014 The role of FDG-PET/CT in 
differentiated thyroid cancer patients with negative iodine-131 whole-body scan and elevated anti-Tg level. Annals of Nuclear Medicine 28 970-979. (https://doi.org/10.1007/s12149-014-0897-7)

Bogsrud TV, Hay ID, Karantanis D, Nathan MA, Mullan BP, Wiseman GA, Kasperbauer JL, Reading CC, Björo T \& Lowe VJ 2011 Prognostic value of $18 \mathrm{~F}$-fluorodeoxyglucose-positron emission tomography in patients with differentiated thyroid carcinoma and circulating antithyroglobulin autoantibodies. Nuclear Medicine Communications 32 245-251. (https://doi.org/10.1097/ MNM.0b013e328343a742

Chiovato L, Latrofa F, Braverman LE, Pacini F, Capezzone M, Masserini L, Grasso L \& Pinchera A 2003 Disappearance of humoral thyroid autoimmunity after complete removal of thyroid antigens. Annals of Internal Medicine 139 346-351. (https://doi. org/10.7326/0003-4819-139-5_Part_1-200309020-00010)

Chung JK, Park YJ, Kim TY, So Y, Kim SK, Park DJ, Lee DS, Lee MC \& Cho BY 2002 Clinical significance of elevated level of serum antithyroglobulin antibody in patients with differentiated thyroid cancer after thyroid ablation. Clinical Endocrinology 57 215-221. (https://doi.org/10.1046/j.1365-2265.2002.01592.x)

Deeks JJ, Macaskill P \& Irwig L 2005 The performance of tests of publication bias and other sample size effects in systematic reviews of diagnostic test accuracy was assessed. Journal of Clinical Epidemiology 58 882-893. (https://doi.org/10.1016/j. jclinepi.2005.01.016)

Dong MJ, Liu ZF, Zhao K, Ruan LX, Wang GL, Yang SY, Sun F \& Luo XG 2009 Value of ${ }^{18 F-F D G-P E T / P E T-C T ~ i n ~ d i f f e r e n t i a t e d ~ t h y r o i d ~}$ carcinoma with radioiodine-negative whole-body scan: a metaanalysis. Nuclear Medicine Communications 30 639-650. (https://doi. org/10.1097/MNM.0b013e32832dcfa7)

Durante C, Tognini S, Montesano T, Orlandi F, Torlontano M, Puxeddu E, Attard M, Costante G, Tumino S, Meringolo D, et al. 2014 Clinical aggressiveness and long-term outcome in patients with papillary thyroid cancer and circulating anti-thyroglobulin autoantibodies. Thyroid 24 1139-1145. (https://doi.org/10.1089/ thy.2013.0698)

Glas AS, Lijmer JG, Prins MH, Bonsel GJ \& Bossuyt PM 2003 The diagnostic odds ratio: a single indicator of test performance. Journal of Clinical Epidemiology 56 1129-1135. (https://doi.org/10.1016/ S0895-4356(03)00177-X)

Görges R, Maniecki M, Jentzen W, Sheu SN, Mann K, Bockisch A \& Janssen OE 2005 Development and clinical impact of thyroglobulin antibodies in patients with differentiated thyroid carcinoma during the first 3 years after thyroidectomy. European Journal of Endocrinology 153 49-55. (https://doi.org/10.1530/eje.1.01940)

Hamza TH, van Houwelingen HC \& Stijnen T 2008 The binomial distribution of meta-analysis was preferred to model within-study variability. Journal of Clinical Epidemiology 61 41-51. (https://doi. org/10.1016/j.jclinepi.2007.03.016)

Haugen BR, Alexander EK, Bible KC, Doherty GM, Mandel SJ, Nikiforov YE, Pacini F, Randolph GW, Sawka AM, Schlumberger M, et al. 20162015 American Thyroid Association Management Guidelines for adult patients with thyroid nodules and differentiated thyroid cancer: the American Thyroid Association Guidelines task force on thyroid nodules and differentiated thyroid cancer. Thyroid 26 1-133. (https://doi.org/10.1089/thy.2015.0020)

Hsieh CJ \& Wang PW 2014 Sequential changes of serum antithyroglobulin antibody levels are a good predictor of disease activity in thyroglobulin-negative patients with papillary thyroid carcinoma. Thyroid 24 488-493. (https://doi.org/10.1089/ thy.2012.0611)

Joensuu H \& Ahonen A 1987 Imaging of metastases of thyroid carcinoma with fluorine-18 fluorodeoxyglucose. Journal of Nuclear Medicine 28 910-914.

Kim WG, Yoon JH, Kim WB, Kim TY, Kim EY, Kim JM, Ryu JS, Gong G, Hong SJ \& Shong YK 2008 Change of serum antithyroglobulin antibody levels is useful for prediction of clinical recurrence in thyroglobulin-negative patients with differentiated thyroid carcinoma. Journal of Clinical Endocrinology and Metabolism 93 4683-4689. (https://doi.org/10.1210/jc.2008-0962)

Kim ES, Lim DJ, Baek KH, Lee JM, Kim MK, Kwon HS, Song KH, Kang MI, Cha BY, Lee KW, et al. 2010 Thyroglobulin antibody is associated with increased cancer risk in thyroid nodules. Thyroid 20 885-891. (https://doi.org/10.1089/thy.2009.0384)

Kingpetch K, Pipatrattana R, Tepmongkol S, Sirisalipoch S \& Chaiwatanarat T 2011 Utility of ${ }^{18}$ F-FDG PET/CT in well differentiated thyroid carcinoma with high serum antithyroglobulin antibody. Journal of the Medical Association of Thailand 94 1238-1244.

Kumar A, Shah DH, Shrihari U, Dandekar SR, Vijayan U \& Sharma SM 1994 Significance of antithyroglobulin autoantibodies in differentiated thyroid carcinoma. Thyroid 4 199-202. (https://doi. org/10.1089/thy.1994.4.199)

Lijmer JG, Mol BW, Heisterkamp S, Bonsel GJ, Prins MH, van der Meulen JH \& Bossuyt PM 1999 Empirical evidence of design-related bias in studies of diagnostic tests. JAMA 282 1061-1066. (https://doi. org/10.1001/jama.282.11.1061)

Ozkan E, Soydal C, Araz M, Aras G \& Ibis E 2012 The additive clinical value of 18F-FDG PET/CT in defining the recurrence of disease in patients with differentiated thyroid cancer who have isolated increased antithyroglobulin antibody levels. Clinical Nuclear Medicine 37 755-758. (https://doi.org/10.1097/ RLU.0b013e31825ae77b)

Ozkan E, Aras G \& Kucuk NO 2013 Correlation of 18F-FDG PET/CT findings with histopathological results in differentiated thyroid cancer patients who have increased thyroglobulin or antithyroglobulin antibody levels and negative 131I whole-body scan results. Clinical Nuclear Medicine 38 326-331. (https://doi. org/10.1097/RLU.0b013e318286827b)

Qiu ZL, Wei WJ, Shen CT, Song HJ, Zhang XY, Sun ZK \& Luo QY 2017 Diagnostic performance of ${ }^{18} \mathrm{~F}-\mathrm{FDG}$ PET/CT in papillary thyroid carcinoma with negative 131I-WBS at first postablation, negative Tg and progressively increased TgAb level. Scientific Reports 72849. (https://doi.org/10.1038/s41598-017-03001-7)

Reitsma JB, Glas AS, Rutjes AW, Scholten RJ, Bossuyt PM \& Zwinderman AH 2005 Bivariate analysis of sensitivity and specificity produces informative summary measures in diagnostic reviews. Journal of Clinical Epidemiology 58 982-990. (https://doi. org/10.1016/j.jclinepi.2005.02.022)

Rutter CM \& Gatsonis CA 2001 A hierarchical regression approach to meta-analysis of diagnostic test accuracy evaluations. Statistics in Medicine 20 2865-2884. (https://doi.org/10.1002/sim.942)

Seo JH, Lee SW, Ahn BC \& Lee J 2010 Recurrence detection in differentiated thyroid cancer patients with elevated serum level of antithyroglobulin antibody: special emphasis on using ${ }^{18}$ F-FDG PET/ CT. Clinical Endocrinology 72 558-563. (https://doi. org/10.1111/j.1365-2265.2009.03693.x)

Siegel RL, Miller KD \& Jemal A 2017 Cancer statistics, 2017. CA: A Cancer Journal for Clinicians 67 7-30. (https://doi.org/10.3322/ caac. 21387)

Souza SL, Montalli Da Assumpção LV \& Ward LS 2003 Impact of previous thyroid autoimmune diseases on prognosis of patients with well-differentiated thyroid cancer. Thyroid 13 491-495. (https://doi. org/10.1089/105072503322021160)

Spencer CA, Bergoglio LM, Kazarosyan M, Fatemi S \& LoPresti JS 2005 Clinical impact of thyroglobulin (Tg) and Tg autoantibody method differences on the management of patients with differentiated thyroid carcinomas. Journal of Clinical Endocrinology and Metabolism 90 5566-5575. (https://doi.org/10.1210/jc.2005-0671)

Spencer C, Petrovic I \& Fatemi S 2011 Current thyroglobulin autoantibody (TgAb) assays often fail to detect interfering $\operatorname{TgAb}$ that can result in the reporting of falsely low/undetectable serum Tg IMA values for patients with differentiated thyroid cancer. Journal of (c) 2018 Society for Endocrinology Published by Bioscientifica Ltd. Printed in Great Britain 
Clinical Endocrinology and Metabolism 96 1283-1291. (https://doi. org/10.1210/jc.2010-2762)

Thompson SG 1994 Why sources of heterogeneity in meta-analysis should be investigated. BMJ 309 1351-1355. (https://doi. org/10.1136/bmj.309.6965.1351)

Tsushima Y, Miyauchi A, Ito Y, Kudo T, Masuoka H, Yabuta T, Fukushima M, Kihara M, Higashiyama T, Takamura Y, et al. 2013 Prognostic significance of changes in serum thyroglobulin antibody levels of pre- and post-total thyroidectomy in thyroglobulin antibody-positive papillary thyroid carcinoma patients. Endocrine Journal 60 871-876. (https://doi.org/10.1507/ endocrj.EJ12-0410)
Viedma S, Borrego Dorado I, Rodríguez Rodríguez JR, Navarro González E, Vázquez Albertino R, Fernández López R \& Agudo Martínez A 2011 Use of ${ }^{18} \mathrm{~F}$ FDG-PET in patients with suspicion of recurrent differentiated thyroid cancer by elevated antithyroglobulin antibodies levels and negative ${ }^{131} \mathrm{I}$ scan. Revista Española de Medicina Nuclear e Imagen Molecular 30 77-82. (https://doi.org/10.1016/j. remn.2010.10.012)

Whiting PF, Rutjes AW, Westwood ME, Mallett S, Deeks JJ, Reitsma JB, Leeflang MM, Sterne JA, Bossuyt PM \& QUADAS-2 Group 2011 QUADAS-2: a revised tool for the quality assessment of diagnostic accuracy studies. Annals of Internal Medicine 155 529-536. (https:// doi.org/10.7326/0003-4819-155-8-201110180-00009)

Received in final form 16 March 2018

Accepted 20 March 2018

Accepted Preprint published online 20 March 2018 (c) 2018 Society for Endocrinology Published by Bioscientifica Ltd. 